\title{
A Preliminary Investigation of Ductility-Enhancement Mechanism through In Situ Nanofibrillation in Thermoplastic Matrix Composites
}

\author{
Bhaskar Patham $^{1}$ and M. P. Poornendu Thejaswini ${ }^{2}$ \\ ${ }^{1}$ General Motors Technical Centre India Pvt. Ltd., Vehicle Performance Centers, Vehicle CAE Methods Group, \\ International Tech Park Ltd., Whitefield Road, Bangalore 560 066, India \\ ${ }^{2}$ General Motors Technical Centre India Pvt. Ltd., Vehicle Performance Centers, Materials and IMDS Group, \\ International Tech Park Ltd., Whitefield Road, Bangalore 560 066, India
}

Correspondence should be addressed to Bhaskar Patham; bhaskar.patham@gm.com

Received 29 March 2013; Revised 17 June 2013; Accepted 20 June 2013

Academic Editor: Zhong-Ming Li

Copyright (C) 2013 B. Patham and M. P. P. Thejaswini. This is an open access article distributed under the Creative Commons Attribution License, which permits unrestricted use, distribution, and reproduction in any medium, provided the original work is properly cited.

\begin{abstract}
A preliminary investigation of interrelationships between tensile stress-strain characteristics and morphology evolution during deformation is conducted on a commercially available thermoplastic composite with a low-surface-energy nanofibrillating poly(tetrafluoroethylene) (PTFE) additive. In this class of composites, the deformation-associated nanofibrillation of the lowsurface-energy additive has been hypothesized to provide an additional dissipation mechanism, thereby enhancing the ductility of the composite. This class of composites offers potential for automotive light weighting in exterior and interior body and fascia applications; it is therefore of interest to investigate processing-structure-property interrelationships in these materials. This study specifically probes the interrelationships between the plastic deformation within the matrix and the fibrillation of the lowsurface-energy additive; tensile tests are carried out at two different temperatures which are chosen so as to facilitate and suppress plastic deformation within the matrix polymer. Based on these preliminary investigations, it is noted that PTFE fibrillation acts synergistically with the ductile deformation of the matrix resin resulting in higher strains to failure of the composite; the results also suggest that the mechanism of fibrillation-assisted enhancement of strains to failure may not operate in the absence of matrix plasticity.
\end{abstract}

\section{Introduction}

Thermoplastic matrix composites comprise an attractive class of materials holding high potential for light-weighting applications; these composites are typically designed to combine the ductility, toughness, and processability of thermoplastic matrices, and the stiffness, strength, thermo-mechanical, and other special functionalities of an additive material. Typical reinforcements that are added to polymeric matrices to enhance the thermo mechanical properties-for example, stiffness, strength, and heat-deflection temperatureinclude fibrous fillers (such as glass and carbon fibers), flakes and discs (such as talc), and nanofillers (such as nanoclay platelets). Typically, the addition of a stiffening reinforcement also leads to a compromise of the ductility of the composite; the resulting composite is rendered more brittle (with a lower strain to failure) compared to the unreinforced matrix. The loss of ductility relative to the unfilled polymer may be attributed to the rigid filler hindering certain degrees of freedom of the polymer to reconfigure its state upon being stressed.

While structural members fabricated from reinforced thermoplastics are typically overdesigned in terms of stiffness so as to avoid the onset of yield strains, in most of the service conditions involving semistructural or nonstructural applications of plastics, it would be more optimal to retain the ductility and toughness of the polymeric matrix while at the same time enhancing the stiffness and thermal service 
properties. It is highly desirable, but usually difficult, to simultaneously accomplish these opposing requirements (high stiffness, high strength, and high ductility) within the same material formulation.

To improve the strains-to-failure of the composite, it would be critical to retain-within the composite-one or more of the significant matrix deformation mechanisms which contribute to its ductility. An investigation of tensile deformation of polypropylenes carried out in situ with smallangle X-ray scattering measurements [1] has associated the ductile deformation of semicrystalline polymers with the dual mechanism of cavitation and stable shear of fibrillated spherulites. Clearly if analogous matrix deformation modes were to be preserved in the composite formulation, the resulting composite would display greater ductility, while improving in stiffness due to the rigid fillers.

By contrast, if compositing with rigid fillers results in the loss of these critical matrix deformation modes, then it would be appropriate to include an additive which simulates an analogous fibrillation behavior within the composite to increase its failure strains. One category of fibrillating inclusions that has been studied in this context is liquid crystalline polymers (LCP) - which have been shown to fibrillate during draw. In one implementation involving modification of a polycarbonate matrix with LCP [2], an increase of LCP crystallite size at higher draw ratios was seen to result in an increase of composite stiffness. Another category of additives, which has received increased attention in the context of ductility enhancement recently [3], and which is relevant to the current study, includes low-surface-energy fluoropolymers. For example, poly(tetrafluoroethylene) (PTFE) has a unique set of physical and chemical properties such as low coefficients of friction, thermal stability, chemical inertness, and high toughness; it is well researched for its low-surfaceenergy properties, for "nonstick" applications [4].

PTFE is known to show two low-temperature thermal transitions below its melting point-a first-order transition from triclinic to hexagonal crystalline form at around $19^{\circ} \mathrm{C}$ and another one at $30^{\circ} \mathrm{C}$ [5-7]. The melting peaks for PTFE range from about $320^{\circ} \mathrm{C}$ to about $350^{\circ} \mathrm{C}$ and are dependent on whether it is in nodular form or fibrillated form [3, 4, 8]. While PTFE powders sheared below $19^{\circ} \mathrm{C}$ typically show particle-flow characteristics, imposition of shear at temperatures typically above $30^{\circ} \mathrm{C}$ (but below the melting point of PTFE) has been shown to result in an unwinding of the packed spherulitic morphology leading to fibrillation $[4,9,10]$. More recent studies involving extrusion of PTFE pastes (e.g., refer to Ariawan et al. [9]) also revealed a similar evolution of PTFE fibrils from interconnected PTFE particles under shear.

When added to polymeric matrices during melt processing, the extent of fibrillation is seen to be a function of the strength of flow field and the residence time within the flow field. It has also been shown that PTFE nanofibrillation results in better melt processability, higher melt strength and drawability, and greater melt elasticity in composites with polypropylene or polycarbonate matrices [8, 11-14].

The addition of PTFE to other polymeric matrices with the aim of imparting higher ductility and increasing the strains to failure of the composite is a relatively recent development [3]. It has been hypothesized [3, 15] that the deformation-induced in situ nanofibrillation of PTFE provides an additional dissipative mechanism during deformation of the composite that results in improved ductility.

The promise of these systems is that the composites may now be formulated with the right combination of stiffness and ductility that is optimal to the component design and service conditions, rather than employing formulations which are overdesigned in terms of stiffness. The composition of such novel composites-involving a singleor multiphase polymeric matrix, a rigid structural filler, and a low surface energy PTFE additive, and the synergistic and antagonistic effects of these components in governing the composite performance-provides an interesting system for fundamental investigations of processing-structure-property interrelationships.

Objective. The overall focus of this research was to investigate the interrelationships between the global deformation behavior of this class of composites and its local microstructure evolution as qualified by the state of dispersion and distribution of the low surface energy PTFE additive. This study very specifically aimed to further the understanding on the interrelationships between the plastic deformation within the matrix and the fibrillation behavior; it sought to answer a particular question: does fibrillation (and the subsequent fibrillation-aided ductility enhancement of the composite) occur independently of matrix ductility? This investigation is essential to facilitate better design of composites with fibrillating additives for performance at extremely low temperatures, where the matrix might be rendered brittle. This understanding would also aid in better design of structural composites with high rigid-filler loading-in which the matrix may be prevented from experiencing yield (due to the overdesign for stiffness)-where the fibrillating filler may be added for impact modification. To start addressing this particular question, it was most important in this study to establish conditions which would effectively facilitate or suppress plastic deformation within the matrix. This was accomplished by carrying out tensile tests-on a commercially available thermoplastic matrix composite comprised of a polymer blend matrix reinforced with rigid talc fillers as well as PTFE additive-at two different temperatures: room temperature at which the matrix blend displays ductile behavior and $-40^{\circ} \mathrm{C}$ at which both the components of the matrix blend are in the vicinity of their ductile-brittle transition temperatures and would be expected to display brittle behavior. Quasistatic tensile tests were conducted up to specimen failure; the structure of the fractured surfaces was then probed using scanning electron microscopy (SEM) and differential scanning calorimetry (DSC). Finally, qualitative correlations between the mechanical behavior of the composite and its structure were inferred.

\section{Experimental}

2.1. Material. A commercial grade of HMD-Xenoy was supplied by SABIC India Pvt. Ltd., Vadodara, India. This grade of 
HMD (high modulus ductile) Xenoy is composed of a blend of polycarbonate (PC) and polybutylene terephthalate (PBT) as the matrix; it contains talc for enhancement of stiffness and strength of the matrix and PTFE for retaining and enhancing the ductility of the composite. The formulation and the exact composition of the composite, including the PTFE content, was not evaluated in this study as it is proprietary to SABIC. Further, it is important to note that this investigation did not specifically address the impact of the PTFE content on this mechanism; neither was this study aimed at evaluation of the performance of the material with respect to its specific intended use.

\subsection{Tensile Specimen Preparation and Tensile Testing}

2.2.1. Specimen Preparation. Tensile test specimens conforming to the ISO-527 standard (ISO 527 Type 1A for rigid sheets with a thickness specification of $4.0 \pm 0.2 \mathrm{~mm}$ ) $[16,17]$ were prepared by injection molding carried out at STEER Polymer Science and Diagnostic Centre, Bangalore, India, using a 100 Ton capacity L\&T S Tech-100 machine. An end-gated ISO 527 Type 1A mold with an L\&T LTM 200 mold temperature controller was employed. Prior to injection molding, the composite pellets were dried and dehumidified at $110^{\circ} \mathrm{C}$ for 5 hours in an L\&T LMD 8 vacuum drier. The temperature settings in the various zones of the injectionmolding machine were set as listed in Table 1 . The injectionmolding processing parameters employed for fabrication of the tensile specimens are listed in Table 2. Injection-molding of tensile specimens was carried out at an injection speed (measured as the linear velocity of the melt front within the mold during mold filling) of $20 \mathrm{~mm} / \mathrm{min}$, which translates to a nominal processing shear rate of $28 \mathrm{~s}^{-1}$.

2.2.2. Tensile Testing. Tensile tests were carried out on an Instron Universal Testing Machine (UTM) Instron 5582, equipped with a retractable environmental chamber with a temperature range of $-150^{\circ} \mathrm{C}$ to $600^{\circ} \mathrm{C}$. Quasistatic tensile tests at a nominal initial strain rate of $10^{-5} \mathrm{~s}^{-1}$ were conducted at room temperature and at a low temperature of $-40^{\circ} \mathrm{C}$. Measurement of strains was conducted using a clip-on extensometer with an initial gage length of 1 inch mounted on the gage section of the tensile specimen (and centered with respect to the length of the specimen). Measurement of loads was conducted using a $100 \mathrm{kN}$ load cell mounted on the crosshead. The load and displacement were recorded until specimen failure. The data recorded by the strain gage after the breakage of the specimens was discarded.

The tests at $-40^{\circ} \mathrm{C}$ were carried out within the enclosure of the environmental chamber that was connected to a pressurized liquefied nitrogen tank. The temperature was maintained by automated control of the flow rate of liquefied nitrogen gas into the enclosure. Prior to the tests, the samples were placed between the test fixtures loosely and allowed to equilibrate at $-40^{\circ} \mathrm{C}$ for about $5 \mathrm{~min}$. The test fixtures were tightened, and the strain gage was mounted only after the sample had sufficiently equilibrated at this low temperature; this was done in order to avoid recording of spurious stresses
TABle 1: Processing temperature of injection molding.

\begin{tabular}{lc}
\hline Zones & Temperature $\left({ }^{\circ} \mathrm{C}\right)$ \\
\hline Feed zone & 50 \\
MH1 & 250 \\
MH2 & 260 \\
MH4 & 270 \\
Nozzle & 265 \\
Mold & 70 \\
\hline
\end{tabular}

TABLE 2: Injection-molding processing parameters.

\begin{tabular}{lc}
\hline Injection pressure (bar) & 80 \\
Injection speed (mm/sec) & 20 \\
Holding pressure (bar) & 60 \\
Holding time (sec) & 5 \\
Cooling time (sec) & 5 \\
Back pressure (Bar) & 5 \\
Refilling screw speed (\%) & $40 \%(100 \mathrm{rpm})$ \\
\hline
\end{tabular}

and strains due to confined shrinkage of the tensile specimen and the test fixture.

A few specimens were also subjected to high-rate tensile tests (employing nominal initial strain rates typically in the range of $10^{-1} \mathrm{~s}^{-1}$ ) at $-40^{\circ} \mathrm{C}$. For these tests, however, the strain gage could not be employed due to the significant inertial effects involved; also, the stress-strain data obtained from these tests could not be attributed a high certainty. These tests were conducted mainly to subject the composite to faster deformation kinematics and thereby modify the conditions for deformation-induced microstructure evolution. The data from these tests are currently being analyzed and will be presented in a subsequent report.

An auxiliary question that this study intended to address was the impact of "as processed" microstructure of the undeformed specimens on their tensile stress-strain behavior and strains to failure. To investigate this aspect, in addition to injection molding at $20 \mathrm{~mm} / \mathrm{s}\left(28 \mathrm{~s}^{-1}\right)$, tensile bar specimens were also injection molded at $50 \mathrm{~mm} / \mathrm{s}$ and $80 \mathrm{~mm} / \mathrm{s}$ - with the corresponding nominal processing strain rates of $70 \mathrm{~s}^{-1}$ and $112 \mathrm{~s}^{-1}$. The premise here was that the initial state of dispersion and distribution of the low surface energy fluoropolymer additive within the undeformed specimen (which in turn would be brought about by the processing conditions employed during injection molding) would have a bearing on the fibrillation behavior during tensile deformation. This premise was based on the reports of fibrillation behavior during extensional deformation of composite melts with PTFE additives (cf. [11-14]). The injection-molding flow front offers a strong extensional flow field, and therefore, the injection rates were varied to effectively vary the extensional kinematics experienced by the melt during injection molding, which was further expected to affect how the fluoropolymer additive would fibrillate during subsequent deformation.

Unfortunately, this aspect could not be investigated in complete detail. This was due to the premature failure observed in the majority of the specimens injection molded 
at high strain rates; this is obvious from an inspection of the representative stress-strain curves obtained for the specimens injection molded at the three injection rates shown in Figure 1. All curves overlap in the elastic region of the response, but only the specimen injection molded at the lowest processing strain rate of $28 \mathrm{~s}^{-1}$ during injection molding shows significant plastic deformation before failure. The tensile specimen molded at $28 \mathrm{~s}^{-1}$ breaks at an engineering strain of around 20\%, while those molded at $70 \mathrm{~s}^{-1}$ and $112 \mathrm{~s}^{-1}$ prematurely break at significantly lower strains of $2 \%$ and $1.5 \%$, respectively.

These premature failures were always associated with signatures of macroscopic voids on the fracture surface. These macroscopic features were observable on both halves of the fractured surfaces of the specimens that failed prematurely, and in some cases were big enough to cover a significant portion of the failure surface. These voids were clearly detectable by the naked eye, and the typical elevation between the "hill" and "valley" regions was of the order of $1 \mathrm{~mm}$ or more in some instances. These macroscopic dimensions are clearly several orders of magnitude larger compared to the submicron dimensions that can be typically associated with the deformation-induced voids that are formed during spherulitic voiding of ductile polymers. Therefore, it may be concluded that such voids associated with the premature failure of specimens are processing defects that are present even in the undeformed specimens; clearly these voids are causative of the premature failure rather than the result of deformation. It is understood that such processing-induced voids may be caused due to moisture uptake by the specimen during storage or due to air entrainment during injection molding. In the current scenario, since such defects were observed only in specimens injection molded at high processing strain rates, these voids may be attributed primarily to air entrainment at high melt flow rates during injection molding.

Since the high rate processed specimens did not always fail in the expected fashion, a thorough investigation of whether the flow kinematics imposed during melt processing (and the resulting morphology of the injection-molded samples) has a significant impact on the microstructure evolution and deformation behavior of the specimens could not be carried out. A more rigorous study-with a more controlled injection-molding setup to avoid void formation at high injection rates-to further investigate these rate effects will be carried out in future.

The defects in specimens molded at $70 \mathrm{~s}^{-1}$ and $112 \mathrm{~s}^{-1}$ also resulted in similar scatter in the tensile tests conducted at the low temperature $\left(-40^{\circ} \mathrm{C}\right)$; therefore, the low temperature results obtained with these specimens are not being reported. Nevertheless, the fracture surfaces obtained at room temperature and $-40^{\circ} \mathrm{C}$ for the specimens injection molded at both $28 \mathrm{~s}^{-1}$ (defect free) and $112 \mathrm{~s}^{-1}$ (containing void defects) were employed for microscopy.

2.3. Microscopy. Microstructural analysis was conducted on the fractured surfaces of the specimens tensile tested in the quasistatic mode up to failure at room temperature and at $-40^{\circ} \mathrm{C}$. The intention of the scanning electron microscopy

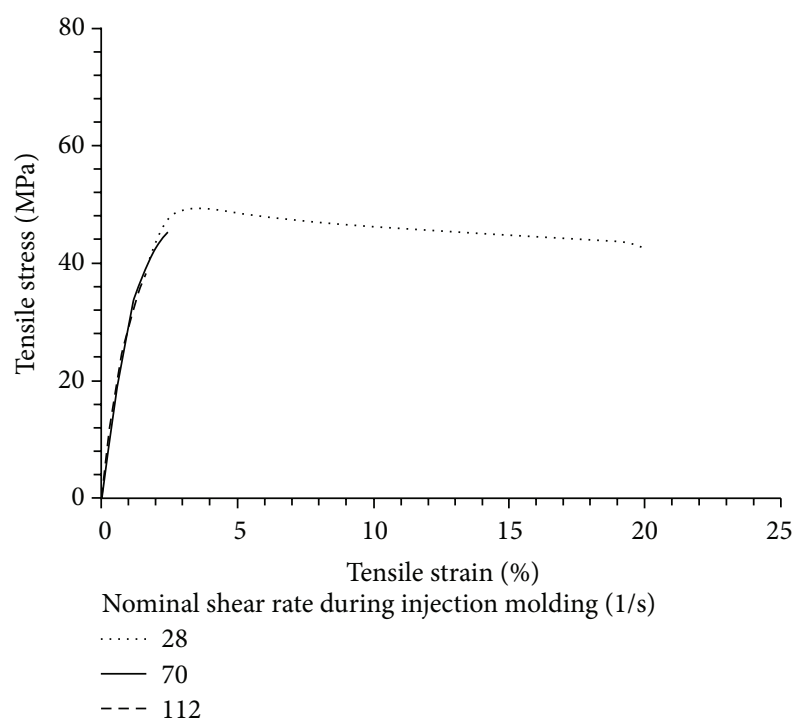

Figure 1: Quasistatic tensile stress-strain curves obtained at room temperature for tensile specimens injection molded at nominal shear rates of $28 \mathrm{~s}^{-1}, 70 \mathrm{~s}^{-1}$, and $112 \mathrm{~s}^{-1}$. The specimens processed at $70 \mathrm{~s}^{-1}$ and $112 \mathrm{~s}^{-1}$ failed prematurely owing to the presence of process-induced macroscopic void defects.

(SEM) analysis was (a) to probe the presence (or absence) of PTFE fibrils and a fibrillation network and (b) to evaluate whether these features connected with fibril dispersion and distribution may be correlated with the global stressstrain characteristics displayed by the specimen. In order to facilitate better resolution of the microstructural features, including nanoscale fibrillated PTFE (if present), a Zeiss 1540XB CrossBeam SEM was used in the field emission mode (FESEM), enabling the use of higher current compared to conventional SEM.

The fracture surfaces were subjected to SEM without any further mechanical treatments to the surface to avoid any alteration of the surface microstructure cumulatively evolved through processing (injection molding) and deformation (tensile testing). For SEM specimen preparation, the fractured half of the tensile bar was held upright using plastic clips, then placed on double sided adhesive carbon tapes for grounding the sample, and then wrapped with aluminum foils to prevent exposure of regions that were not relevant for microscopic analysis. The exposed region (the fractured surface) of the specimen, being nonconductive, was then prepared for SEM environment by depositing a conductive coating of gold-palladium (80/20) on the surface using an SC 7620 Mini Sputter Coater for 150 s.

2.4. Differential Scanning Calorimetry (DSC). DSC was carried out using a Q2000 DSC from TA Instruments. DSC thermograms were generated for the unprocessed pellets and the specimen cut from the fracture surface of the failed tensile bars. Argon gas was used for thermal equilibration within the DSC environment. The samples were characterized in ramp mode with a ramp rate of $10^{\circ} \mathrm{C}$ per min starting from $40^{\circ} \mathrm{C}$ (standby temperature) up to $400^{\circ} \mathrm{C}$. This temperature range 
covered all major primary and secondary transitions of all the polymeric components within the composite (except the lowtemperature primary peaks of PTFE [5-7]). In order to probe the first-order melting transitions of PTFE more sensitively, the DSC experiments were conducted in a "modulated" mode (MDSC); the temperature ramp was superimposed with a temperature modulation with amplitude of $1^{\circ} \mathrm{C}$ for $30 \mathrm{~s}$ imposed every $5 \mathrm{~min}$. This frequency domain characterization, superimposed on the time-domain based standard ramp test, provided a convoluted heat signal, which was then deconvoluted by the TA DSC software into reversible and irreversible portions. The deconvoluted heat curves were expected to provide a significantly more sensitive probe of the PTFE first-order thermal transition compared to the conventional time-domain temperature ramp experiment. Thermograms of the fractured specimens were compared to that of an unprocessed pellet to infer any signatures of microstructure development during deformation.

\section{Results and Discussion}

3.1. Tensile Stress-Strain Behavior. In Figure 2, the quasistatic tensile stress-strain curve measured at room temperature is compared with that measured at $-40^{\circ} \mathrm{C}$ for the defect-free tensile bar specimen injection molded at a nominal shear rate of $28 \mathrm{~s}^{-1}$. Both of the stress-strain curves reported are obtained up to the point of specimen failure. The specimen tested at room temperature showed significant plastic deformation before failure. The stress-strain curve obtained at $-40^{\circ} \mathrm{C}$ displays a higher initial slope (higher modulus) as well as higher strength compared to that measured at room temperature. On the other hand, the failure strain observed for the specimen at $-40^{\circ} \mathrm{C}$ is significantly lower compared to that observed in room temperature testing; the specimen fails before onset of yield.

"Control" tests-involving the composite system without the low surface energy fluoropolymer additive-could not be conducted. This was because the system studied was a commercially formulated composite, and an equivalent commercially available composite with exactly the same composition but without the fibrillating additive could not be identified.

However, there is data available at room temperature on other equivalent (but not exact) systems from the SABIC-IP Xenoy family which contain a polycarbonate-polyester blend as the matrix with mineral fillers. For example, for the room temperature test, the recorded tensile yield strength of about $50 \mathrm{MPa}$ is comparable to the values reported in literature for commercially available PC/PBT blends containing mineral fillers and PTFE additives [15]. The secant modulus of $2.5 \mathrm{Gpa}$ evaluated at $1 \%$ strain for this specimen is also comparable to (only slightly lesser than) the typical values reported in the literature for commercially available PC/PBT blends containing mineral fillers and significantly higher than the values of moduli reported for unfilled PC/PBT blends [15]. The elongation strain-to-failure of $20 \%$ for this specimen falls short of the values (up to $100 \%$ ) reported in literature [15] for commercially available PC/PBT blends containing PTFE

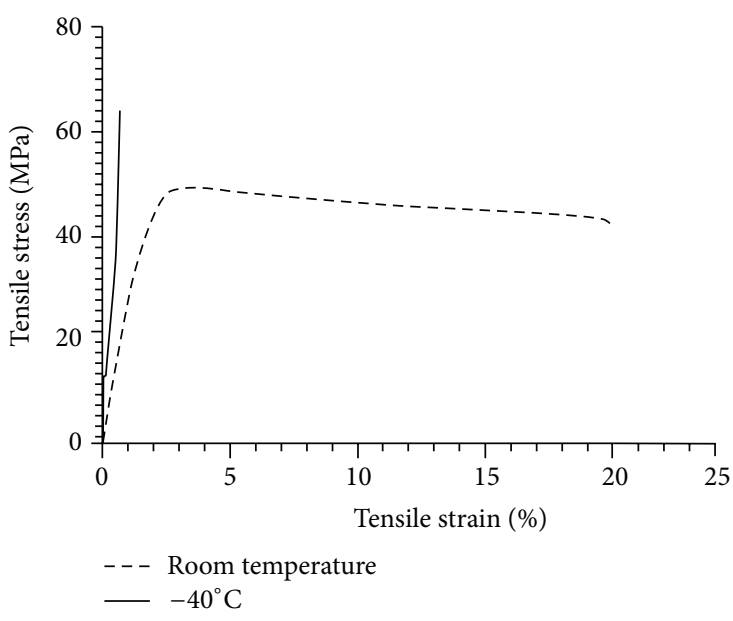

FIGURE 2: Quasistatic tensile stress-strain curve measured at room temperature compared with that measured at $-40^{\circ} \mathrm{C}$.

additives. The difference may be tied to the exact nature and content of mineral fillers as well as the amount of PTFE additive in the batch of the composite used in this study, compared to that within the batches for which these values were reported in [15]. Notwithstanding these differences, these strains-to-failure are still significantly larger than the values-ranging from $3 \%[18]$ to $10 \%[15,19]$-reported in the literature for mineral-filled PC/PBT blends without the fibrillating additives; fibrillation, if present in the fractured specimen, may therefore be unambiguously correlated with the increased strain to failure.

Unlike in case of the room temperature specimen, control data on strains to failure at low temperatures for equivalent mineral filled PC/PBT blends without fibrillating fillers was not available in the literature. In the absence of such baseline information, it would not be possible to unambiguously discern whether the response of the composite at $-40^{\circ} \mathrm{C}$ is dominated by the brittleness of the matrix or whether fibrillation (if present in the fracture surface of the specimen) has any impact on the strain to failure. However, as discussed in the subsequent, the lack of such baseline information does not significantly alter the conclusions from this study, since the microscopy of the specimen fracture surfaces reveals stark contrasts in fibrillation behavior at room temperature and $-40^{\circ} \mathrm{C}$.

3.2. Morphology-Property Interrelationships. FESEM micrographs of the fracture surfaces of the tensile specimens quasistatic tensile tested at $-40^{\circ} \mathrm{C}$ are shown in Figures 3 and 4 , respectively, for specimens injection molded at nominal shear rates of $112 \mathrm{~s}^{-1}$ and $28 \mathrm{~s}^{-1}$. In both micrographs, the failure patterns in the form of rough wavy topography are clearly visible. Another typical feature is the presence of faceted topography, with a typical wavelength of 2 microns. Flake like structures, of the order of 2 microns, appear to project out of the fracture surfaces; in some areas, these projections are adjacent to voids, suggesting a pullout of particulate inclusions, also of the order of about 2 microns. All these 


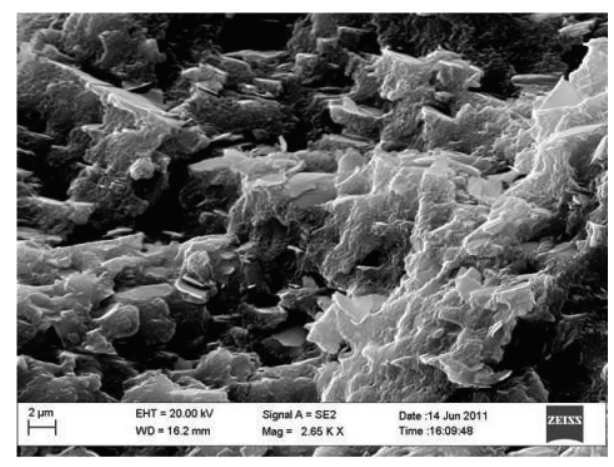

FIGURE 3: FE SEM of quasistatic tensile fractured surface obtained at $-40^{\circ} \mathrm{C}$ for the tensile specimen injection molded at a nominal shear rate of $112 \mathrm{~s}^{-1}$.

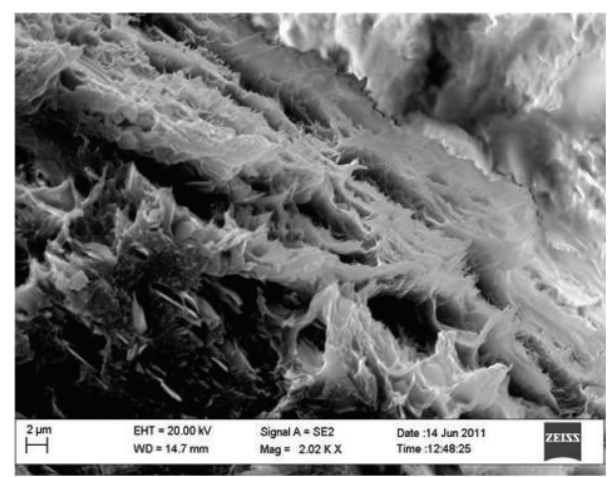

FIGURE 4: FE SEM of quasistatic tensile fractured surface obtained at $-40^{\circ} \mathrm{C}$ for the tensile specimen injection molded at a nominal shear rate of $28 \mathrm{~s}^{-1}$.

features appear to be related to talc fillers, given the flaky nature of the particulate inclusions, and the rectangular shape of the voids associated with particle pullout. The "faceted" morphology is indicative of inadequate deformation of the matrix and is consistent with the brittle failure characteristic at the low temperature. While the faceted morphology was predominant in the failure surfaces of the specimens tested at $-40^{\circ} \mathrm{C}$, another less prominent feature, which was more observable in the fractured surface of the tensile specimen processed at $28 \mathrm{~s}^{-1}$, was the presence of intermittent "floral" patterns pulled out in the tensile direction, indicating matrix deformation (refer to Figure 4). However, PTFE fibrillation was not discernible in any of the failure surfaces generated at $-40^{\circ} \mathrm{C}$.

FESEM micrographs of the fracture surfaces of the tensile specimens quasistatic tensile tested at room temperature are shown in Figures 5 and 6, respectively, for the specimens injection molded at nominal shear rates of $112 \mathrm{~s}^{-1}$ and $28 \mathrm{~s}^{-1}$. Just as in failure surfaces obtained with the specimens tested at $-40^{\circ} \mathrm{C}$, the features associated with flaky fillers may be clearly observed in these micrographs also. However, the "floral" topography associated with matrix deformation is much more pronounced in the failure surfaces generated in the room temperature tested specimens. More importantly, in contrast to low temperature tested samples, a fibrillated

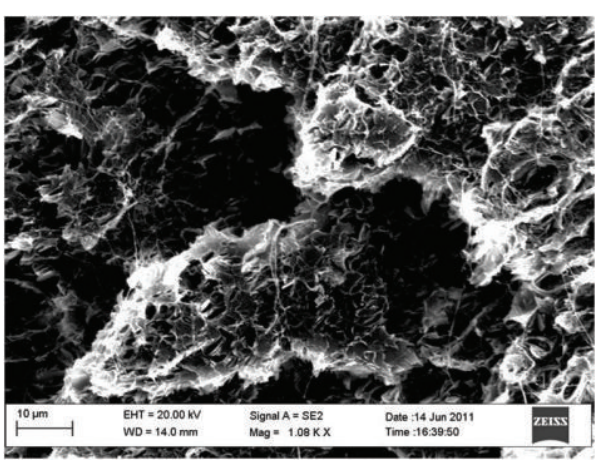

FIGURE 5: FE SEM of quasistatic tensile fractured surface obtained at room temperature $\left(24^{\circ} \mathrm{C}\right)$ for the tensile specimen injection molded at a nominal shear rate of $112 \mathrm{~s}^{-1}$.

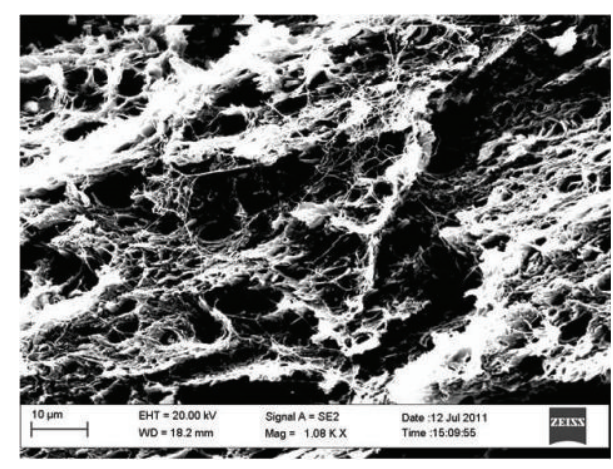

FIGURE 6: FE SEM of quasistatic tensile fractured surface obtained at room temperature $\left(24^{\circ} \mathrm{C}\right)$ for the tensile specimen injection molded at a nominal shear rate of $28 \mathrm{~s}^{-1}$.

network is clearly discernible in both Figures 5 and 6. Since no other component of the composite shows fibrillation, this feature may be unambiguously attributed to PTFE fibrillation.

Further evidence of deformation-associated fibrillation of PTFE during the ductile failure of the composite may be seen in the MDSC thermograms plotted in Figure 7. In this figure, the nonreversible heat input during MDSC of the unprocessed HMD Xenoy composite pellet is compared with those of undeformed tensile specimen and the fractured region of a room temperature quasistatic tensile tested specimen; both tensile specimens were injection molded at $28 \mathrm{~s}^{-1}$. A prominent first order transition at a temperature of about $350^{\circ} \mathrm{C}$ is observable only in the ductile-fractured region of the room temperature tensile tested specimen, indicating the evolution of PTFE fibrillation and orientation during the deformation. The quasistatic failed specimen also shows a small peak in the vicinity of $100^{\circ} \mathrm{C}$ which can be attributed to the presence of moisture in the specimen; this is not surprising, given the hygroscopic nature of polycarbonate, which is one of the components of the polymer blend matrix. This detail is not of significant importance in the context of fibrillation of the low surface energy fluoropolymer additive (none of the thermal transitions of the latter are expected around $100^{\circ} \mathrm{C}$ ). 


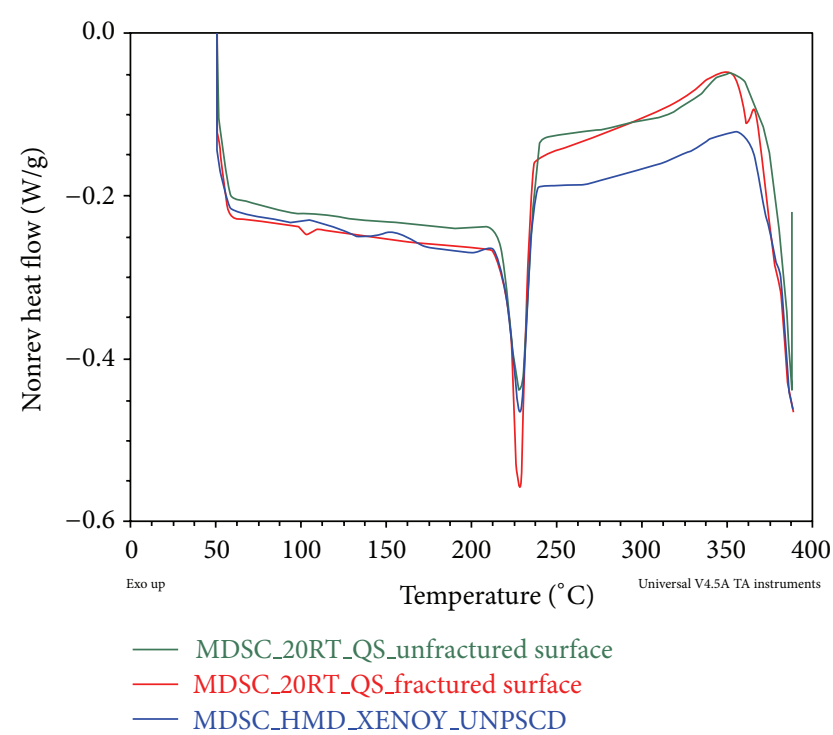

FIgURE 7: Nonreversible heat flow MDSC thermogram of unprocessed as-received HMD Xenoy composite pellet compared with those of undeformed tensile specimen and the fractured region of a room temperature quasistatic tensile-tested tensile specimen; both tensile specimens were injection molded at a nominal shear rate of $28 \mathrm{~s}^{-1}$.

3.3. Discussion. In room temperature tests, the tensile specimen processed at $112 \mathrm{~s}^{-1}$ which had a void defect discernible on the failure surface, failed at a significantly lower strain compared to that processed at $28 \mathrm{~s}^{-1}$ which did not have any defects. While the specimen processed at $112 \mathrm{~s}^{-1}$ did not display significant yield before failing, the specimen processed at $28 \mathrm{~s}^{-1}$ showed significant plastic deformation before failure. Both the specimens however displayed fibrillated network on the failure surfaces. On the other hand, the significantly lower strain to failure before onset of yield observed for the specimens at $-40^{\circ} \mathrm{C}$ is consistent with the fact that both the components of the matrix blend are expected to display brittle characteristics. None of the specimens tested at $-40^{\circ} \mathrm{C}$ displayed fibrillation.

Based on the previous observations, two trends may be noticed.

First is the clear contrast in terms of fibrillation behavior at the two test temperatures. At room temperature, both the components of the matrix polymer blend-PC and PBTare significantly above their brittle-ductile-transition temperature. While both PC and PBT are below their respective glass transition temperatures, $\mathrm{PC}$ significantly so $\left(T_{g, \mathrm{PC}}=\right.$ $150^{\circ} \mathrm{C} ; T_{g, \mathrm{PBT}} \sim 60^{\circ} \mathrm{C}$ ), they still display significant plastic deformation before failure at room temperature. The generally accepted brittle-ductile-transition temperatures for PC and $\mathrm{PBT}$ are $-25^{\circ} \mathrm{C}$ and $-40^{\circ} \mathrm{C}$, respectively [20]. Therefore, at $-40^{\circ} \mathrm{C}$, the matrix phase is expected to be brittle and not undergo plastic deformation before failure. Comparing the strain to failure of the specimen processed at $28 \mathrm{~s}^{-1}(20 \%)$ with that reported for equivalent mineral filled PC/PBT composites without fibrillating fillers (3-10\%) [15, 18, 19], the increase in composite ductility may be unambiguously correlated with the presence of fibrillation. If fibrillation was observed in the failed specimens tested at $-40^{\circ} \mathrm{C}$, an analogous conclusion would have been difficult to arrive since information about strains to failure of equivalent composite systems without fibrillating fillers was not available at $-40^{\circ} \mathrm{C}$. However, no fibrillation was observed in the specimens tested at $-40^{\circ} \mathrm{C}$. Clearly, the response of these composites was dominated by the brittleness of the matrix. More importantly, the suppression of matrix ductility seems to have prevented the onset of fibrillation in the composite system. Clearly, there appears to be a synergistic effect between the tendency of PTFE to fibrillate and the mechanical behavior of the matrix resin in governing the resulting ductility of the composite.

Second is the observation of fibrillation in roomtemperature-failed specimens irrespective of whether the composite failed after showing enhanced ductility (as in the specimen processed at $28 \mathrm{~s}^{-1}$ ) or prematurely (as in the specimen processed at $112 \mathrm{~s}^{-1}$ ). This observation is a little counter intuitive, because the global stress-strain behavior observed for the specimen processed at $112 \mathrm{~s}^{-1}$ does not display pronounced plastic deformation. However, since the specimen was tested at room temperature, it is understood that the matrix blend has a tendency to deform in a plastic fashion, even though the specimen prematurely failed at a relatively low strain. Two inferences may be made from this observation: firstly, as long as matrix ductility is not suppressed, fibrillation may be observed in the specimen, irrespective of the strain to failure. And secondly, that fibrillation must be associated with localized matrix plasticity; localized plasticity in the absence of specimen-level necking may occur due to unequal load transfer across the specimen cross-section. Of course at $-40^{\circ} \mathrm{C}$, even these local plasticity effects would be suppressed.

\section{Summary}

Room temperature tensile tests reveal ductile behavior of the composite, especially the defect-free specimens processed at $28 \mathrm{~s}^{-1}$. But the tensile tests at $-40^{\circ} \mathrm{C}$ result in significantly lower strains-to-failure, driven by the brittleness of the PCPBT blend matrix, both the components of which were at or below their ductile-brittle transition temperatures at $-40^{\circ} \mathrm{C}$. The low-temperature tested samples, in which the $\mathrm{PC} / \mathrm{PBT}$ blend matrix was rendered brittle, did not show any traces of PTFE fibrillation. The room temperature failure which occurs in a ductile mode is clearly associated with PTFE nanofibrillation. The nonreversible portion of the heat flow in MDSC thermogram also contains the signature of deformation-associated PTFE fibrillation and orientation during the ductile failure of the specimen, in consistency with the micrographs.

At room temperature, when both the components of the matrix blend display the tendency for plastic deformation before failure, the increase in composite ductility (relative to equivalent composite systems without the fibrillating filler) may be unambiguously correlated with the presence of fibrillation. At or below the ductile-brittle transition 
temperature of the matrix resin, the mechanical response of the composite is dominated by the brittleness of the matrix. In other words, the suppression of matrix ductility seems to prevent the onset of fibrillation in the composite system. Clearly, there appears to be a synergistic effect between the tendency of PTFE to fibrillate and the mechanical behavior of the matrix resin in governing the resulting ductility of the composite.

\section{Open Questions and Future Work}

As discussed earlier, an auxiliary investigation on the impact of "as processed" microstructure of the undeformed specimens (brought about by variation of the strengths of the extensional flow field imposed by varying the injection rate) on their tensile stress-strain behavior and strain to failure could not be investigated in complete detail; this was due to the premature failures observed in the majority of the specimens injection molded at high strain rates (due to the presence of process-induced voids). A more rigorous studywith a more controlled injection-molding setup to avoid void formation at high injection rates-to further investigate these rate effects will be carried out in future. This study, combined with detailed quantification of the PTFE surface area on the micrographs of fractured specimens, may result in a better understanding of correlations between the failure strain and the extent of PTFE fibrillation.

In order to more fully understand the structure property relationships in this composite system, similar investigations are required at more testing temperatures, for example at temperatures where one or both of the components of the blend may be at or above their respective glass transition temperatures.

The presence of fibrillation in the prematurely failed specimen in room temperature studies (which was processed at $112 \mathrm{~s}^{-1}$ and which had a void) is an important finding and presents the motivation to investigate further (1) the effect of localized plastic deformation within the matrix (particularly in scenarios involving uneven stress transfer across the specimen cross section) on the fibrillation and ductility-enhancement characteristics and (2) the conditions, especially in terms of a localized threshold plastic strain within the matrix, associated with the onset of fibrillation. These studies are underway and will be presented in a subsequent report.

As noted earlier, a few specimens were also subjected to high-rate tensile tests (employing strain rates typically in the range of $10^{-1} \mathrm{~s}^{-1}$ ) at $-40^{\circ} \mathrm{C}$ so as to subject the composite to faster deformation kinematics and thereby modify the conditions for deformation-induced microstructure evolution. The data from these tests are currently being analyzed and will be presented in a subsequent report.

\section{Conflict of Interests}

The authors hereby declare that they have no conflict of interests.

\section{Acknowledgment}

The authors acknowledge several constructive remarks from the reviewers.

\section{References}

[1] X. C. Zhang, M. F. Butler, and R. E. Cameron, "The ductilebrittle transition of irradiated isotactic polypropylene studied using simultaneous small angle X-ray scattering and tensile deformation," Polymer, vol. 41, no. 10, pp. 3797-3807, 2000.

[2] Q. Lin, J. Jho, and A. F. Yee, "Effect of drawing on structure and properties of a liquid crystalline polymer and polycarbonate insitu composite," Polymer Engineering and Science, vol. 33, no. 13, pp. 789-798, 1993.

[3] S. Bandyopadhyay, R. Kamalakaran, S. Gupta, R. Mazumdar, B. Sathapathy, and A. K. Ghosh, "Fibrillation of teflon in polymer matrices," in Proceedings of the International Conference on Advances in Polymer Technology (APT '10), pp. 71-74, Kochi, India, February 2010.

[4] S. Ebnesajjad, Fluoroplastics, Volume 1: Non-Melt Processible Fluoroplastics, Plastics Design Library, New York, NY, USA, 2000.

[5] C. W. Burn and E. R. Howells, "Opinion," Nature, vol. 174, pp. 569-573, 1954.

[6] C. A. Sperati and H. W. Starkweather, "Fluorine-containing polymers. II. Polytetrafluoroethylene," Fortschritte der Hochpolymeren-Forschung, vol. 2, no. 4, pp. 465-495, 1961.

[7] S. Mazur, "Paste extrusion of poly (tetrafluoroethylene). Fine powders," in Polymer Powder Technology, M. Narkis, Ed., pp. 441-481, John Wiley, New York, NY, USA, 1995.

[8] M. A. B. M. Ali, S. Nobukawa, and M. Yamaguchi, "Morphology development of polytetrafluoroethylene in a polypropylene melt (IUPAC technical report)," Pure and Applied Chemistry, vol. 83, no. 10, pp. 1819-1830, 2011.

[9] A. B. Ariawan, S. Ebnesajjad, and S. G. Hatzikiriakos, "Paste extrusion of polytetrafluoroethylene (PTFE) fine powder resins," Canadian Journal of Chemical Engineering, vol. 80, no. 6, pp. 1153-1165, 2002.

[10] J. G. Drobny, Technology of Flouropolymers, CRC Press, Boca Raton, Fla, USA, 2008.

[11] A. B. M. M. Ali, K. Okamoto, M. Yamaguchi, T. Kasai, and A. Koshirai, "Rheological properties for polypropylene modified by polytetrafluoroethylene," Journal of Polymer Science B, vol. 47, no. 20, pp. 2008-2014, 2009.

[12] T. Murata, J. Takimoto, and K. Koyama, "Melt property and extrusion foaming processability of polypropylene blended with polytetrafluoroethylene," Zairyo/Journal of the Society of Materials Science, Japan, vol. 45, no. 12, pp. 1300-1305, 1996.

[13] T. Kurose, T. Takahashi, and K. Koyama, "Uniaxial elongational viscosity of FEP/ a small amount of PTFE blends," Nihon Reoroji Gakkaishi, vol. 31, no. 4, pp. 195-200, 2003.

[14] T. Kurose, T. Takahashi, M. Sugimoto, T. Taniguchi, and K. Koyama, "Uniaxial elongational viscosity of PC/ a small amount of PTFE blend," Nihon Reoroji Gakkaishi, vol. 33, no. 4, pp. 173182, 2005.

[15] L. M. Sherman, "Plastics technology," February 2009, http:// www.ptonline.com/articles/novel-pc-polyester-blends-combine-stiffness-and-ductility.

[16] ISO 527-1, "Plastics-determination of tensile properties-part 1: general principles," 2012, within ISO Standards Catalogue 83. 080. 01: Plastics in general. 
[17] ISO 527-2, "Plastics-determination of tensile properties-part 2: test conditions for moulding and extrusion plastics," 2012, within ISO Standards Catalogue 83. 080. 01: Plastics in general.

[18] "Xenoy Resin 6370 Datasheet," 2013, SABIC Innovative Plastics, http://www.sabic-ip.com/gepapp/eng/weather/weatherhtml? sltRegionList $=1002002000$ \&sltPrd $=1002003020 \&$ sltGrd= $1002011525 \&$ sltUnit $=0 \&$ sltModule $=$ DATASHEETS $\&$ sltVersion=Internet\&sltType $=$ Online .

[19] “Xenoy Resin XD1647 Datasheet," 2013, SABIC Innovative Plastics, http://www.sabic-ip.com/gepapp/eng/weather/weatherhtml?sltRegionList $=1002002001 \&$ sltPrd $=1002003066 \&$ sltGrd $=$ $1002012923 \&$ sltUnit $=0$ \&sltModule $=$ DATASHEETS\&sltVer sion=Internet\&sltType=Online.

[20] Omnexus Polymer Selector, "Ductile/brittle transition temperature of plastics," http://www.datapointlabs.com/ExpCapShowDetails.asp?InstId=34. 

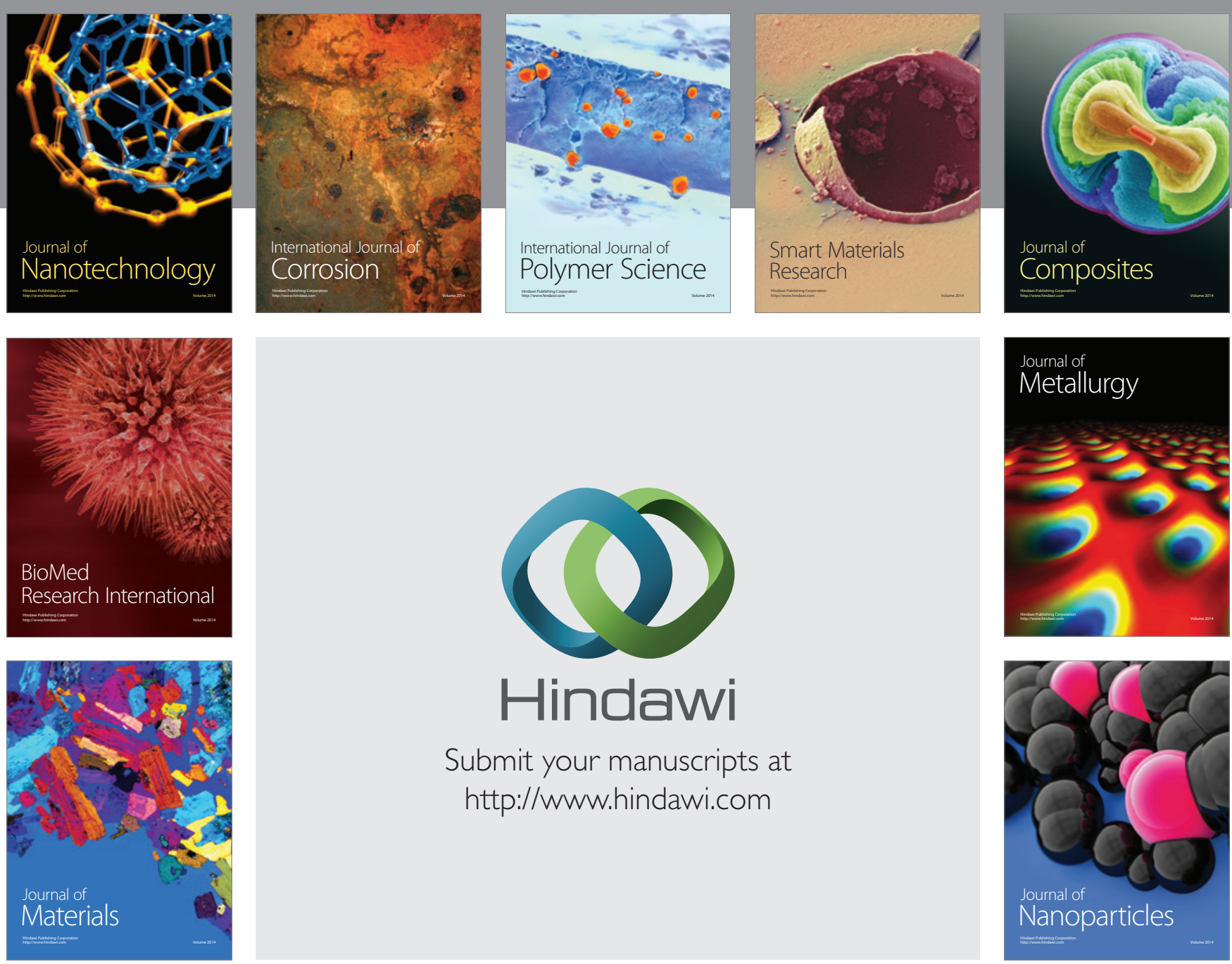

Submit your manuscripts at http://www.hindawi.com
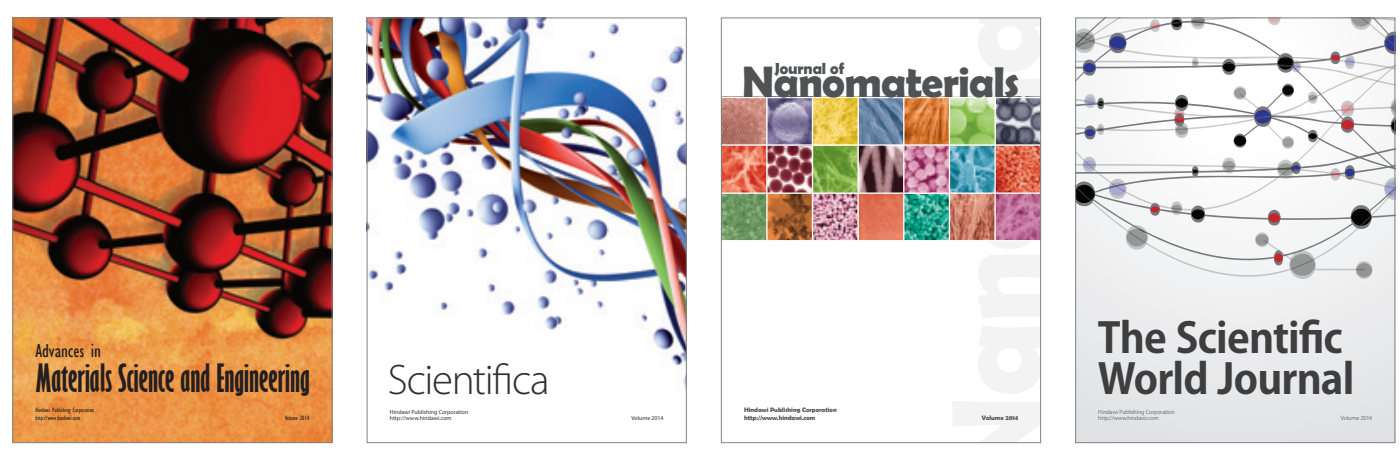

\section{The Scientific World Journal}
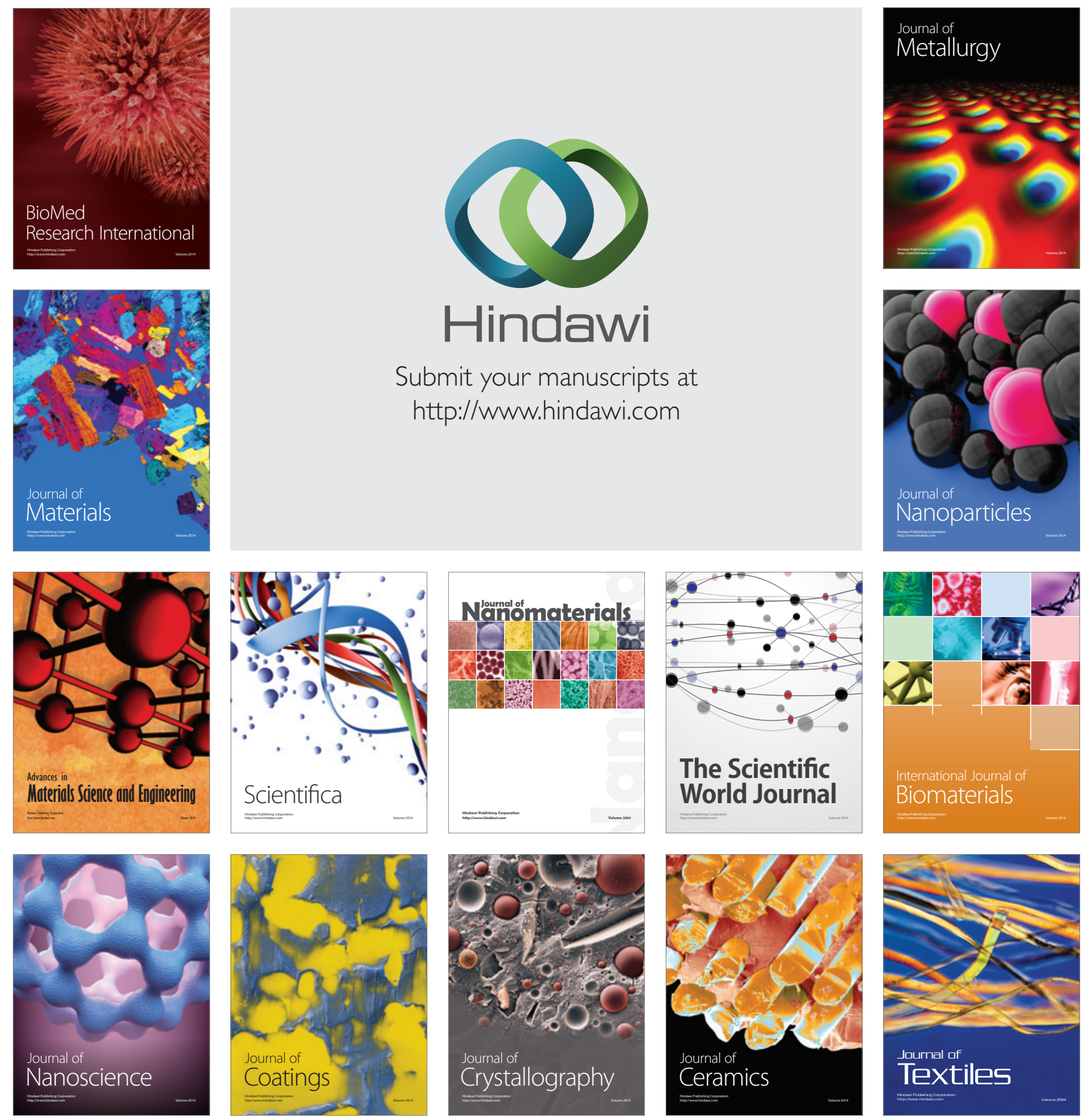